\title{
Erratum: Quantum interference control of localized carrier distributions in the Brillouin zone [Phys. Rev. B 100, 075203 (2019)]
}

\author{
Perry T. Mahon ๑, Rodrigo A. Muniz, and J. E. Sipe \\ (Received 1 April 2020; published 26 June 2020)
}

DOI: 10.1103/PhysRevB.101.239902

We thank R. Binder for bringing to our attention that the spin-orbit parameter $(2 \hbar \lambda)$ we implemented for $\mathrm{WSe}_{2}$ in our paper is incorrect. Consequently, the figures produced using the parameters listed in that paper are not accurate for this transitionmetal dichalcogenide. Importantly, the qualitative nature of the distribution of electronic excitations in the Brillouin zone (BZ) mediated by the various photon absorption processes are unchanged since $\mathrm{WSe}_{2}$ was only implemented as a test bed to illustrate the localization of such excitations. However, due to the altered band structure arising from the different sets of parameters, we set the excitation energy now to be $\hbar \Omega=1.8 \mathrm{eV}$. The corrected and modified parameters we now adopt for the WSe $\mathrm{Wre}_{2}$ listed below in Table I.

TABLE I. Model parameters for $\mathrm{WSe}_{2}$.

\begin{tabular}{lcc}
\hline \hline$\hbar \Xi$ & $2 \hbar \lambda$ & $\hbar \Delta$ \\
\hline $3.9 \AA \mathrm{eV}$ & $0.46 \mathrm{eV}$ & $1.6 \mathrm{eV}$ \\
\hline \hline
\end{tabular}

We display the updated plots associated with the correct parameters below. Notable is that the degree to which $2+3$ photon absorption is able to mediate more localized excitations in the BZ than $1+2$ photon absorption, specifically for colinearly polarized applied fields, is reduced compared to the previous parameter set. This is a consequence of the larger spin-orbit coupling implemented there, which resulted in a larger energy spacing between the relevant conduction bands, and ultimately allowed for a greater degree of localized excitations. 


\section{ELECTRONIC DISTRIBUTION IN THE BRILLOUIN ZONE}
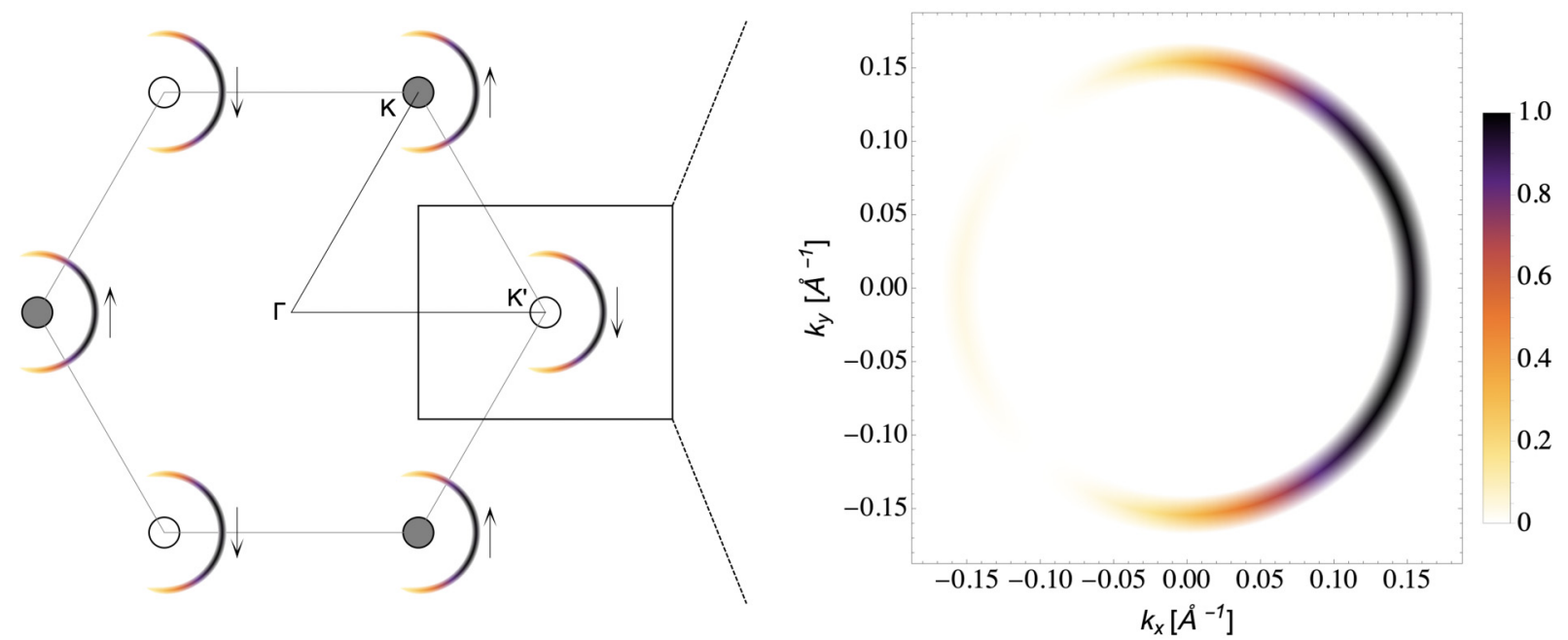

FIG. 1. Distribution of injected carriers in crystal momentum space from an optical probe facilitating $1+2$ photon absorption; the first Brillouin zone is composed of a single pair of $\boldsymbol{K}$ (filled circles) and $\boldsymbol{K}^{\prime}$ (empty circles) points.

\section{A. Colinearly polarized incident fields}

\section{1. $1+2$ absorption}

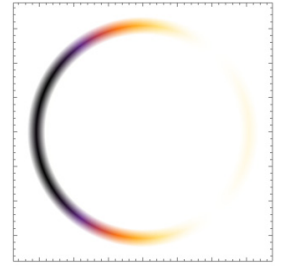

(a) $\Delta \phi_{12}=\pi / 2$

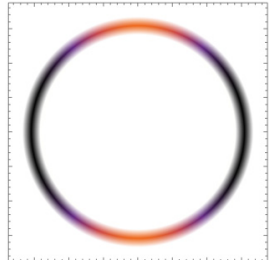

(b) $\Delta \phi_{12}=0$

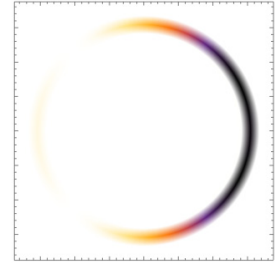

(c) $\Delta \phi_{12}=3 \pi / 2$

FIG. 2. Dependence of $\frac{d}{d t}\left\langle n_{c}(\boldsymbol{k})\right\rangle_{1+2}$ on $\Delta \phi_{12}$ for fields of frequency $\omega$ and $2 \omega$ both polarized along $\hat{\boldsymbol{x}}$.

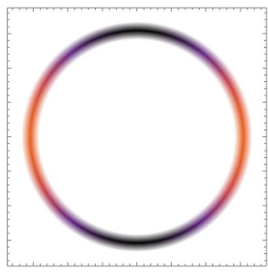

(a) $\frac{d}{d t}\left\langle n_{c}(\boldsymbol{k})\right\rangle_{1}$

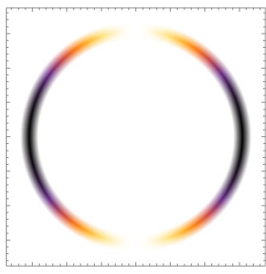

(b) $\frac{d}{d t}\left\langle n_{c}(\boldsymbol{k})\right\rangle_{2}$

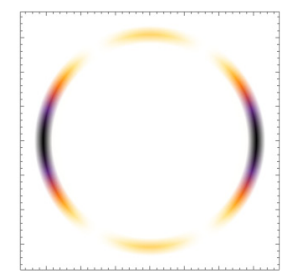

(c) $\frac{d}{d t}\left\langle n_{c}(\boldsymbol{k})\right\rangle_{3}$

FIG. 3. BZ resolved carrier injection rates arising from single color absorption for fields polarized along $\hat{\boldsymbol{x}}$. 


\section{2. $2+3$ absorption}

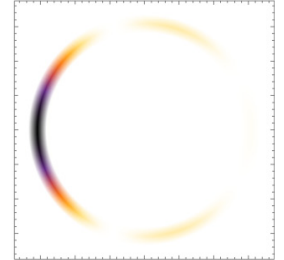

(a) $\Delta \phi_{23}=\pi / 2$

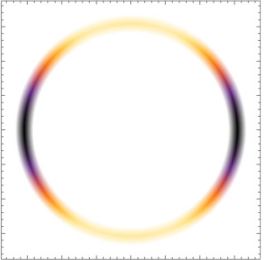

(b) $\Delta \phi_{23}=\pi$

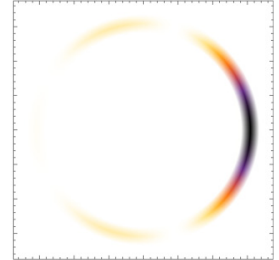

(c) $\Delta \phi_{23}=3 \pi / 2$

FIG. 4. Dependence of $\frac{d}{d t}\left\langle n_{c}(\boldsymbol{k})\right\rangle_{2+3}$ on $\Delta \phi_{23}$ for fields of frequency $\omega$ and $3 \omega / 2$ both polarized along $\hat{\boldsymbol{x}}$.

\section{3. $1+3$ absorption}

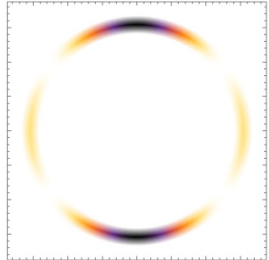

(a) $\Delta \phi_{13}=0$

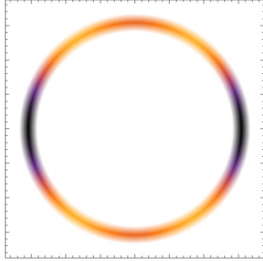

(b) $\Delta \phi_{13}=\pi / 2$

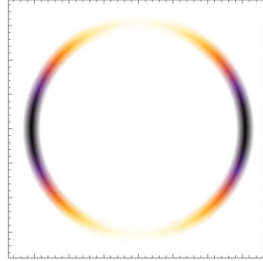

(c) $\Delta \phi_{13}=\pi$

FIG. 5. Dependence of $\frac{d}{d t}\left\langle n_{c}(\boldsymbol{k})\right\rangle_{1+3}$ on $\Delta \phi_{13}$ for fields of frequency $\omega$ and $3 \omega$ both polarized along $\hat{\boldsymbol{x}}$.

\section{B. Cross-linearly polarized incident fields}

\section{1. $1+2$ absorption}

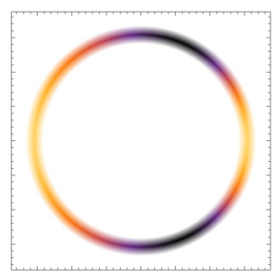

(a) $\boldsymbol{K}, \Delta \phi_{12}=\frac{\pi}{2}$

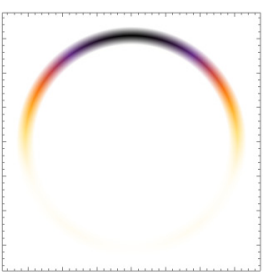

(b) $\boldsymbol{K}, \Delta \phi_{12}=\pi$

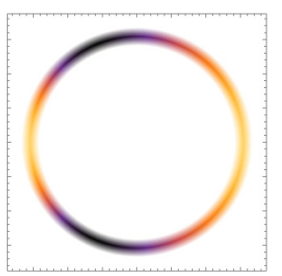

(c) $\boldsymbol{K}, \Delta \phi_{12}=\frac{3 \pi}{2}$

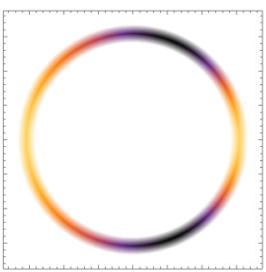

(d) $\boldsymbol{K}^{\prime}, \Delta \phi_{12}=\frac{\pi}{2}$

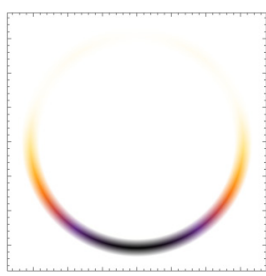

(e) $\boldsymbol{K}^{\prime}, \Delta \phi_{12}=\pi$

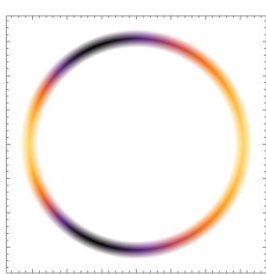

(f) $\boldsymbol{K}^{\prime}, \Delta \phi_{12}=\frac{3 \pi}{2}$

FIG. 6. Dependence of $\frac{d}{d t}\left\langle n_{c}(\boldsymbol{k})\right\rangle_{1+2}$ on $\Delta \phi_{12}$ for fields orientated according to $\hat{\boldsymbol{e}}_{\omega}=\hat{\boldsymbol{y}}$ and $\hat{\boldsymbol{e}}_{2 \omega}=\hat{\boldsymbol{x}}$.

\section{2. $2+3$ absorption}
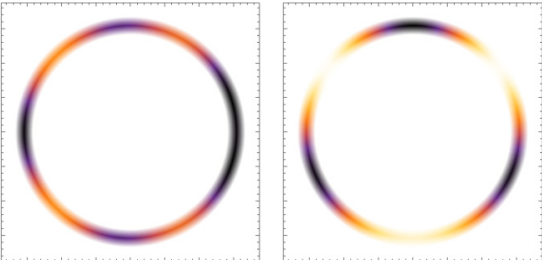

(a) $\boldsymbol{K}, \Delta \phi_{23}=\pi / 2$

(b) $\boldsymbol{K}, \Delta \phi_{23}=\pi$

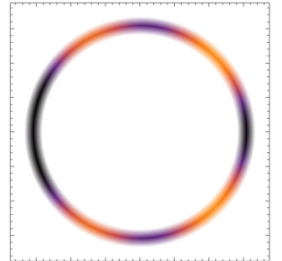

(c) $\boldsymbol{K}, \Delta \phi_{23}=\frac{3 \pi}{2}$
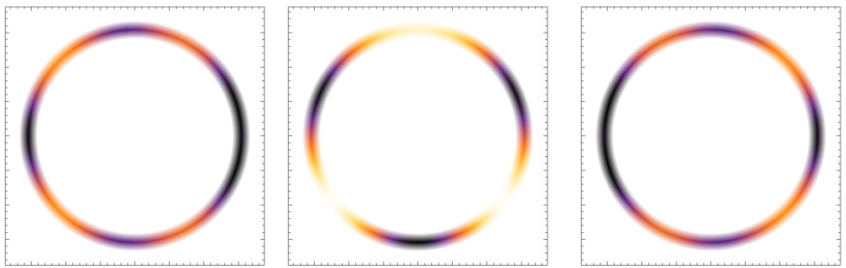

(d) $\boldsymbol{K}^{\prime}, \Delta \phi_{23}=\frac{\pi}{2}$

(f) $\boldsymbol{K}^{\prime}, \Delta \phi_{23}=\frac{3 \pi}{2}$

FIG. 7. Dependence of $\frac{d}{d t}\left\langle n_{c}(\boldsymbol{k})\right\rangle_{2+3}$ on $\Delta \phi_{23}$ for fields orientated according to $\hat{\boldsymbol{e}}_{\omega}=\hat{\boldsymbol{x}}$ and $\hat{\boldsymbol{e}}_{3 \omega / 2}=\hat{\boldsymbol{y}}$. 


\section{3. $1+3$ absorption}

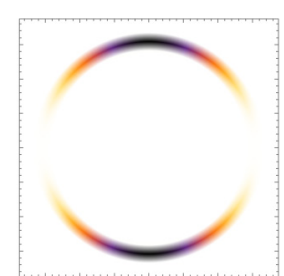

(a) $\boldsymbol{K}, \Delta \phi_{13}=\frac{\pi}{2}$

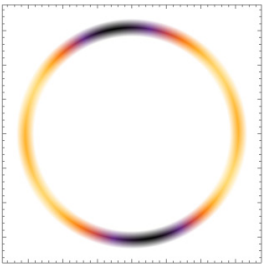

(b) $\boldsymbol{K}, \Delta \phi_{13}=\pi$

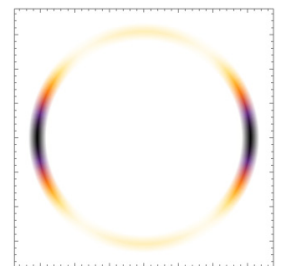

(c) $\boldsymbol{K}, \Delta \phi_{13}=\frac{3 \pi}{2}$

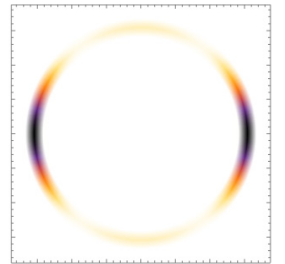

(d) $\boldsymbol{K}^{\prime}, \Delta \phi_{13}=\frac{\pi}{2}$

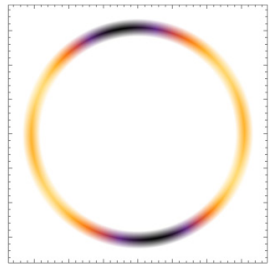

(e) $\boldsymbol{K}^{\prime}, \Delta \phi_{13}=\pi$

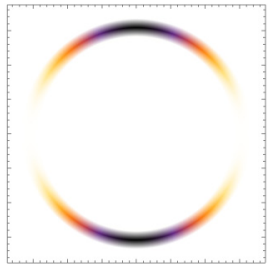

(f) $\boldsymbol{K}^{\prime}, \Delta \phi_{13}=\frac{3 \pi}{2}$

FIG. 8. Dependence of $\frac{d}{d t}\left\langle n_{c}(\boldsymbol{k})\right\rangle_{1+3}$ on $\Delta \phi_{13}$ for fields orientated according to $\hat{\boldsymbol{e}}_{\omega}=\hat{\boldsymbol{y}}$ and $\hat{\boldsymbol{e}}_{3 \omega}=\hat{\boldsymbol{x}}$.

\section{Circularly polarized incident optical fields}

\section{Equal helicities}

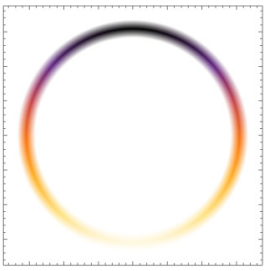

(a) $\Delta \phi_{12}=0$

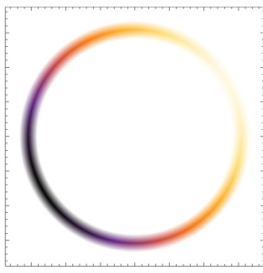

(b) $\Delta \phi_{12}=2 \pi / 3$

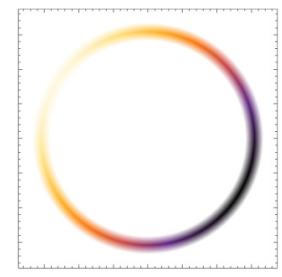

(c) $\Delta \phi_{12}=4 \pi / 3$

FIG. 9. Dependence of $\frac{d}{d t}\left\langle n_{c}(\boldsymbol{k})\right\rangle_{1+2}$ on $\Delta \phi_{12}$ for fields of frequency $\omega$ and $2 \omega$ both circularly polarized in $\hat{\boldsymbol{e}}_{+}$.

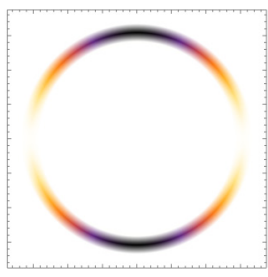

(a) $\Delta \phi_{13}=0$

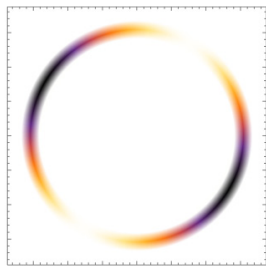

(b) $\Delta \phi_{13}=2 \pi / 3$

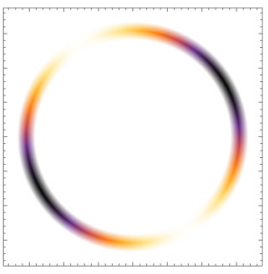

(c) $\Delta \phi_{13}=4 \pi / 3$

FIG. 10. Dependence of $\frac{d}{d t}\left\langle n_{c}(\boldsymbol{k})\right\rangle_{1+3}$ on $\Delta \phi_{13}$ for fields of frequency $\omega$ and $3 \omega$ both circularly polarized in $\hat{\boldsymbol{e}}_{+}$.

\section{Opposite helicities}

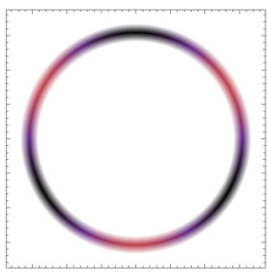

(a) $\Delta \phi_{12}=0$

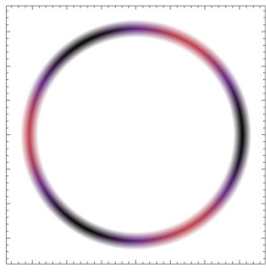

(b) $\Delta \phi_{12}=\pi / 2$

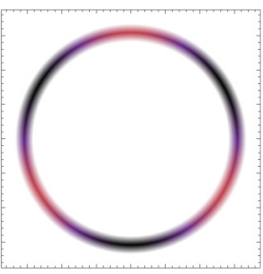

(c) $\Delta \phi_{12}=\pi$

FIG. 11. Dependence of $\frac{d}{d t}\left\langle n_{c}(\boldsymbol{k})\right\rangle_{1+2}$ on $\Delta \phi_{12}$ for fields orientated according to $\hat{\boldsymbol{e}}_{\omega}=\hat{\boldsymbol{e}}_{+}$and $\hat{\boldsymbol{e}}_{2 \omega}=\hat{\boldsymbol{e}}_{-}$. 


\section{FREQUENCY DEPENDENCE OF INJECTION COEFFICIENTS}

\section{A. Carrier injection coefficients}
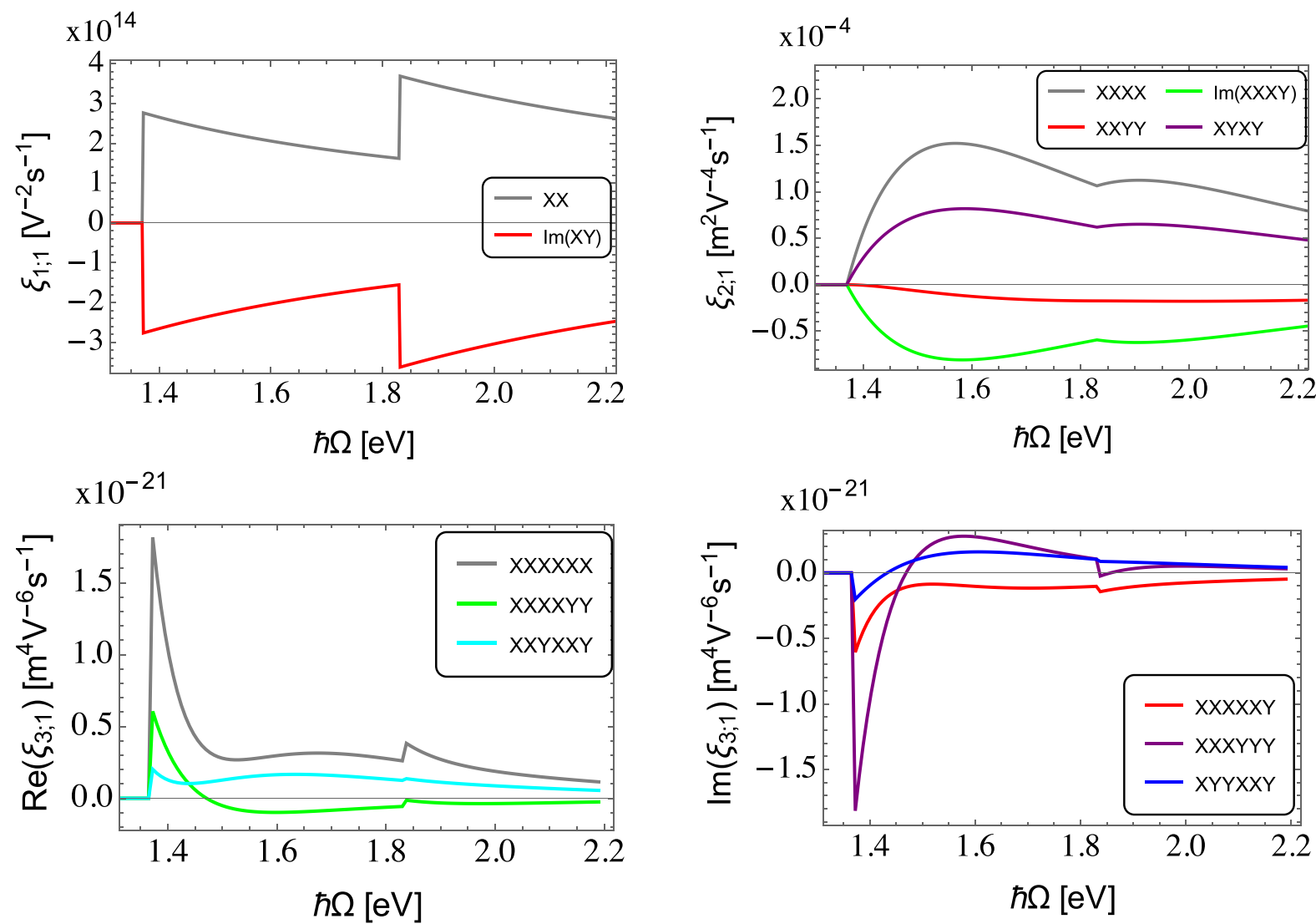

FIG. 12. Excitation energy dependence of the independent components of the carrier injection response tensor for single color photon absorption processes. We plot the nonzero components about the $\boldsymbol{K}$ valley.
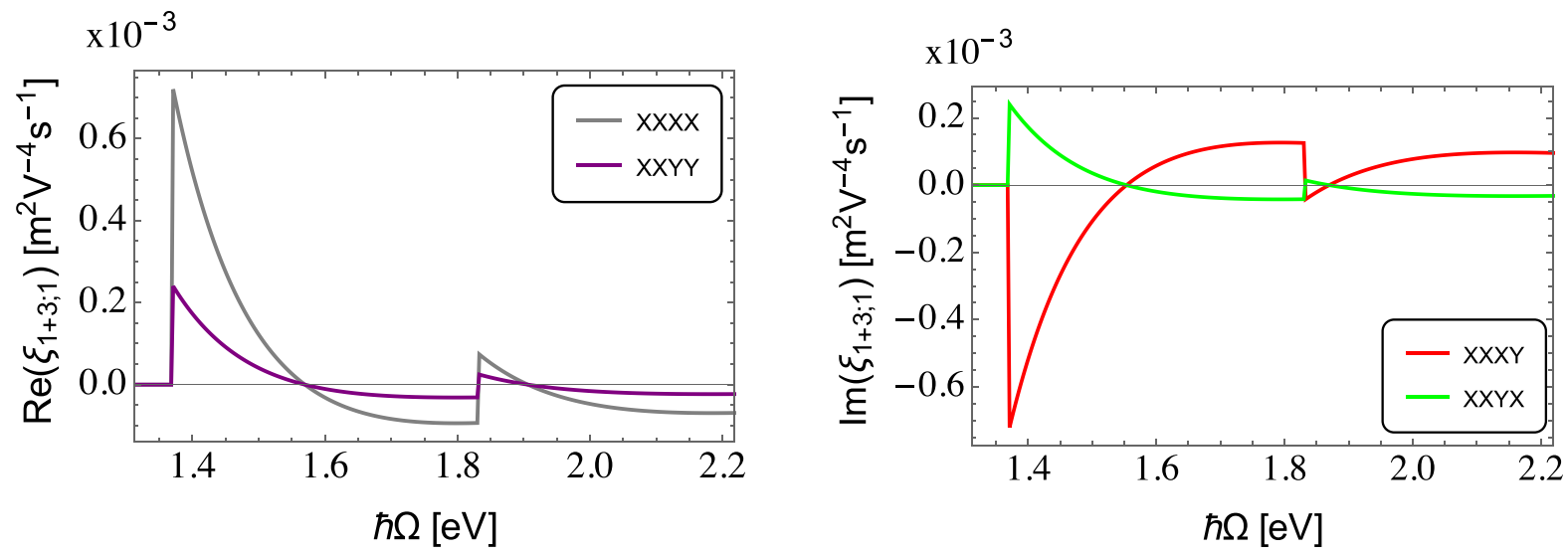

FIG. 13. Excitation energy dependence of the independent components of the carrier injection response tensor arising from the interference of photon absorption processes. We plot the nonzero components about the $\boldsymbol{K}$ valley. 


\section{B. Current injection coefficients}
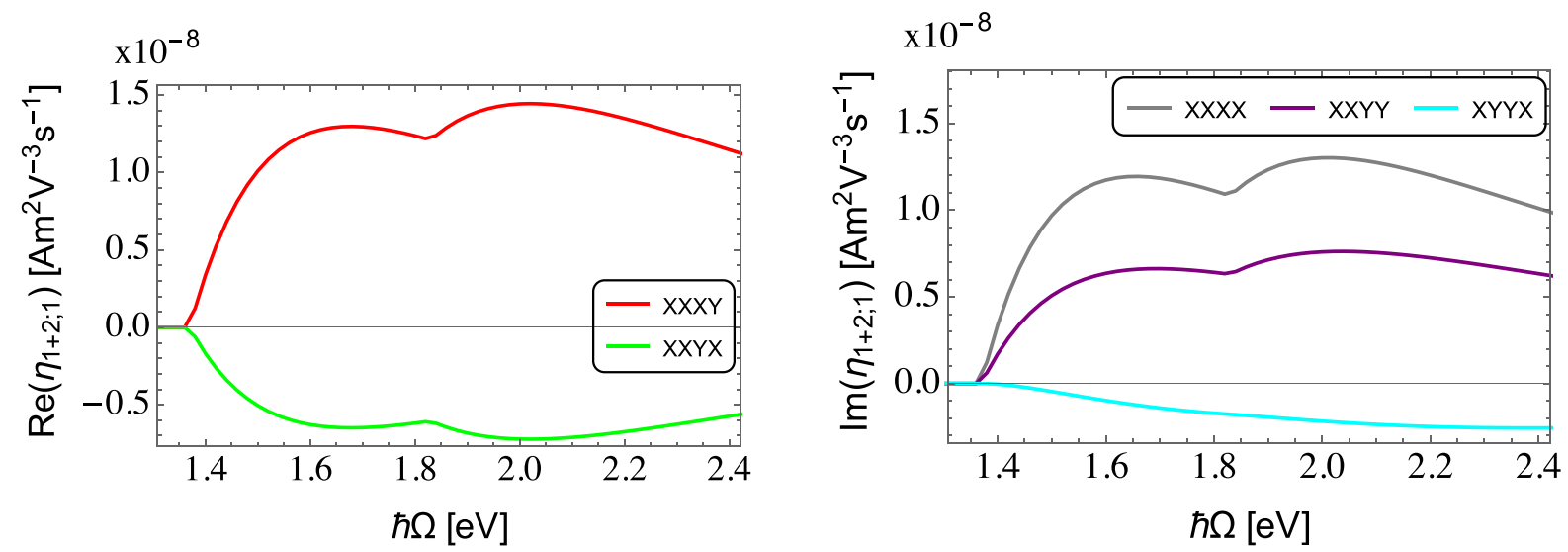

FIG. 14. Excitation energy dependence of the independent components of the current injection response tensor for $1+2$ photon absorption processes. We plot components about a single valley $\boldsymbol{K}$ and omit vanishing components.
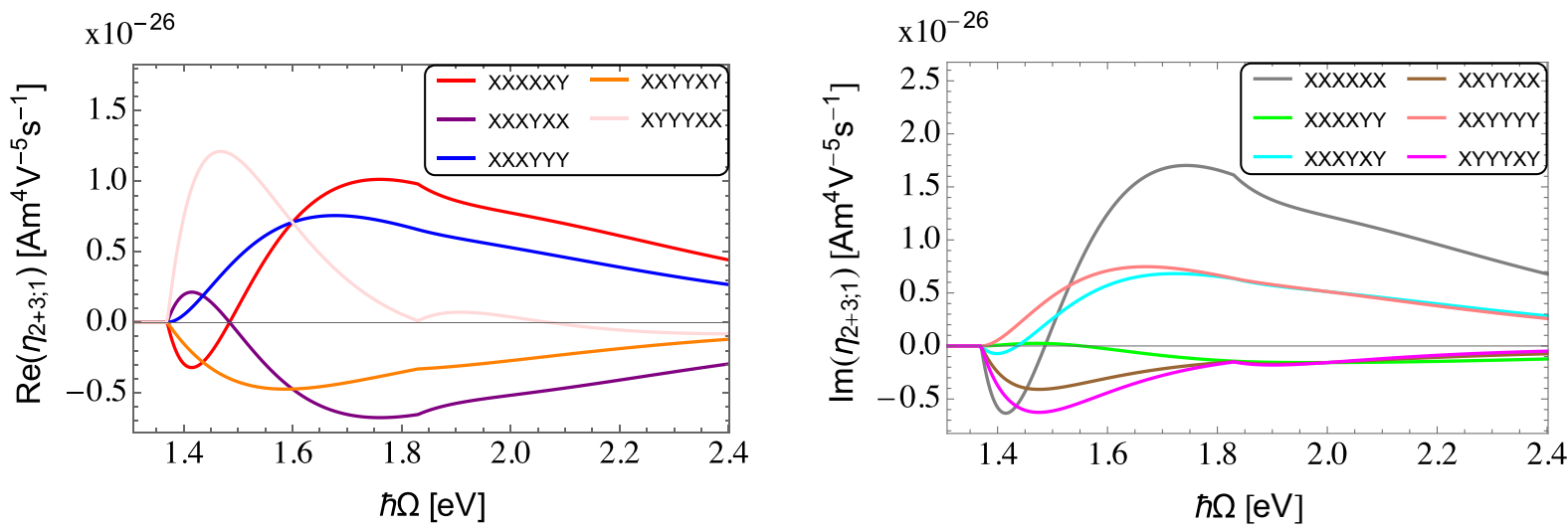

FIG. 15. Excitation energy dependence of the independent components of the current injection response tensor for $2+3$ photon absorption processes. We plot components about a single valley $\boldsymbol{K}$ and omit vanishing components.

\section{Swarm velocities}

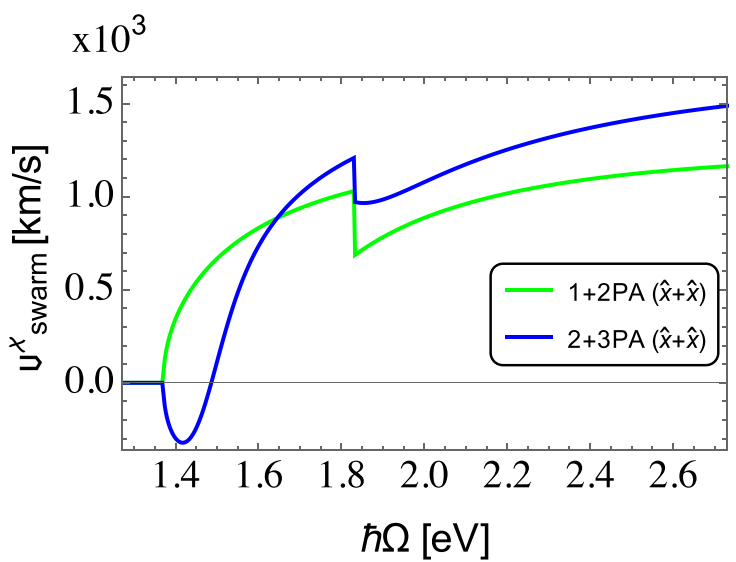

(a) Co-linearly polarized incident fields.

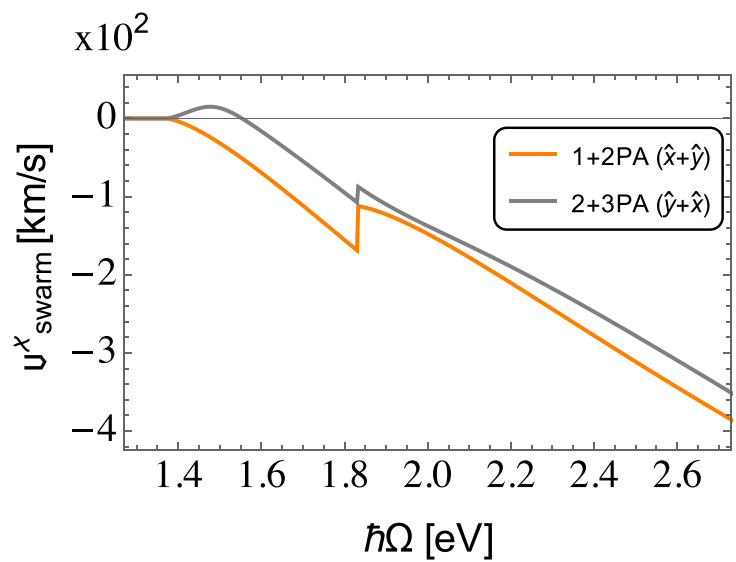

(b) Cross-linearly polarized incident fields.

FIG. 16. Swarm velocities for co- and cross-linearly polarized optical fields. The relative polarizations of the fields are indicated in the legend, and combinations of polarizations that lead to a vanishing swarm velocity in the $\pm \hat{\boldsymbol{x}}$ direction are omitted. 